\title{
Subcellular localization of Mycobacterium leprae-specific phenolic glycolipid (PGL-I) antigen in human leprosy lesions and in M. leprae isolated from armadillo liver
}

\author{
J. BodDINGIUS* and H. DiJKMAN \\ Department of Dermatology, Research Laboratory, Medical Faculty, Erasmus University, Rotterdam, The Netherlands
}

(Received 8 June 1990; accepted 26 June 1990)

\begin{abstract}
Phenolic glycolipid (PGL-I), an antigen specific to Mycobacterium leprae, was localized subcellularly in M. leprae residing in human skin, in $M$. leprae isolated from armadillo liver ('isolated $M$. leprae') and outside $M$. leprae in human lepromatous skin. For a quantitative localization of PGL-I sites, specimens, including skin segments stored for 6 years in glutaraldehyde, were embedded in hydrophilic Lowicryl $\left(\mathbf{K}_{4} M\right)$ resin for ultrathin sectioning. Ultracryosections and Araldite sections of comparable specimens were used for comparison of localization results. A monoclonal antibody (F 47-21-3) directed to antigenic oligosaccharide of PGL-I was employed as primary antibody in immunogold labelling of ultrathin sections. $\mathbf{K}_{\mathbf{4}} \mathbf{M}$-immunogold methods gave very satisfactory quantitive gold-labelling of PGL-I. The localization of PGL-I by this method partially corresponded with sites detectable in both ultracryosections and the qualititatively superior Araldite sections, but new sites were also localized. Cell walls in human $M$. leprae and in isolated $M$. leprae possessed many PGL-I sites, particularly in dividing organisms. PGL-I or its antigenic oligosaccharide was also found, to a lesser extent, in the bacterial cytoplasm. Capsules discernible around part of isolated $M$. leprae cells displayed heavy PGL-I labelling, sometimes clearly confined to a zone distant from the cell wall. Extrabacterial PGL-I in $M$. leprae-infected human skin was encountered (1) in phagolysosomes and cytoplasm proper of dermal macrophages containing $M$. leprae, and (2) intra- and extracellularly in epidermal areas where basal cells harboured $M$. leprae in untreated multibacillary patients.
\end{abstract}

\section{Introduction}

Phenolic glycolipids (PGLs), found in mycobacterial envelopes, are mycosides possessing unusual methylated sugars (Barksdale \& Kim, 1977; Minnikin, 1982). PGL$\mathrm{I}$, the predominant PGL antigen in Mycobacterium leprae (Hunter \& Brennan, 1981, 1983; Hunter et al., 1982; Fujiwara et al., 1984) may play a role in the intracellular persistence of $M$. leprae in human macrophages by scavenging toxic oxygen metabolites of antimicrobial systems of the host cell (Neill \&-Klebanoff, 1988). PGL-I or its antigenic oligosaccharide are used on a small scale

\footnotetext{
* Address to which all correspondence concerning the manuscript and requests for reprints should be sent: Dr J. Boddingius, Department of Dermatology, Erasmus University, c/o PO Box 733, 4200 AS Gorinchem, The Netherlands.
}

Abbreviations: GA, glutaraldehyde; GAMG 10 , gold (10 nm) labelled goat anti-mouse IgG; mAb, monoclonal antibody; PBS, phosphatebuffered saline $\left(0.05 \mathrm{~g} \mathrm{KCl}, 2 \mathrm{~g} \mathrm{NaCl}, 0.95 \mathrm{~g} \mathrm{NaH}_{2} \mathrm{PO}_{4} .2 \mathrm{H}_{2} \mathrm{O}\right.$ and $2.84 \mathrm{~g} \mathrm{Na}_{2} \mathrm{HPO}_{4}$ in $250 \mathrm{ml}$ distilled water; $\mathrm{pH} \mathrm{7.3);} \mathrm{PF,} \mathrm{paraformalde-}$ hyde; PGL-I, phenolic glycolipid-I. in the serodiagnosis of leprosy and in the monitoring of the infection (Cho et al., 1983, 1986; Young \& Buchanan, 1983; Agis et al., 1988; Chanteau et al., 1989).

Neither the surface location of PGL-I in M. leprae, indicated by immunofluorescence (Young et al., 1984), nor possibly persisting pockets of extrabacterial PGL-I antigen in infected tissues (Boddingius \& Dijkman, $1989 a$ ), have yet been investigated in detail in a quantitatively satisfactory way. The localization of either peribacterial (surface) or intrabacterial (nonsurface) PGL-I, and of small amounts of extrabacterial PGL-I, all require immuno-electron microscopic methods. Our previous electron microscopic studies have dealt with the histopathology of human leprosy lesions (Boddingius, 1974, 1976, 1977; Boddingius et al., 1989), histophysiology of lepromatous mouse nerves (Boddingius, 1984) and, recently, the locations of defined antigens - an $M$. leprae-specific lipid antigen and protein $(36 \mathrm{kDa} ; 65 \mathrm{kDa})$ antigens (Boddingius \& Dijkman, $1989 a, b)$.

Three procedural steps are essential for immuno- 
electron microscopic investigation of any $M$. leprae lipid or protein antigen not previously studied. Firstly, ultracryosectioning and immunogold labelling of 'nonfixed' material containing the antigen(s) is done, using $M$. leprae isolated from the liver of the nine-banded armadillo (Dasypus novemcinctus Linn.). Secondly, very mild to 'hard' fixation techniques for the ultrastructural preservation of bacterial cells or of tissues infected by $M$. leprae are employed prior to ultracryosectioning and immunogold labelling. In this step, the possible influence of fixation on immunogenicity of the antigen(s) should be checked by counting gold-labelled cells. Thirdly, $M$. leprae or infected tissues, after treatment with fixatives, are embedded in resin(s) in order to achieve optimal subcellular immuno-localization of antigens. The first two steps were partially carried out by us in pilot studies on $36 \mathrm{kDa}$ and $65 \mathrm{kDa}$ protein antigens (Boddingius \& Dijkman, 1989b). All three steps were partially realized in studies of PGL-I using hydrophobic Araldite resin as an embedding medium (Boddingius \& Dijkman, 1989a). With our new Araldite-immunogold method we obtained accurate subcellular localization of PGL-I in $M$. leprae and extrabacterially in human leprosy skin. However, the method was considered merely qualitative in character, and tissue embedding in Lowicryl was thought to be essential for any quantitative evaluation of PGL-I locations (Boddingius \& Dijkman, 1989a).

In the present study, Lowicryl $\mathrm{K}_{\mathbf{4}} \mathrm{M}$ was tested for satisfactory quantitative immunogold localization of PGL-I. Localization was carried out in $M$. leprae isolated from armadillo liver (henceforth called isolated $M$. leprae), in $M$. leprae residing in human skin, and extrabacterially in human skin and armadillo liver. Isolated $M$. leprae were dispersed in gelatin blocks for processing. The human skin segments used were part of a stock of skin and nerve biopsies which had been stored for 6 years in glutaraldehyde. Lowicryl $K_{4} M$ is a hydrophilic resin which allows good access by antibodies to antigen sites. The resin can be used for tissue embedding both at low $\left(-35^{\circ} \mathrm{C}\right)$ and higher temperatures (up to $60^{\circ} \mathrm{C}$ ). Our work indicates the efficiency of $\mathrm{K}_{4} \mathrm{M}$ embedding for the quantitatively satisfactory demonstration both of PGL-I in $M$. leprae and of extrabacterial PGL-I in human lepromatous skin. An efficient cold-room processing and embedding scheme designed by us renders Lowicryl-immunogold localization of PGL-I widely applicable and also accessible in (sub)tropical countries where leprosy is endemic.

\section{Methods}

Armadillo-derived Mycobacterium leprae ('isolated $M$. leprae'). M. leprae were isolated from infected armadillo liver following standard- ized WHO/IMMLEP procedures (World Health Organization, 1980). The isolated $M$. leprae $\left(10^{10} M\right.$. leprae per $\mathrm{ml}$ PBS) were stored at $-70^{\circ} \mathrm{C}$ (Royal Tropical Institute, Amsterdam, The Netherlands), and transported in liquid nitrogen to Rotterdam for use in the experiments described here.

M. leprae-gelatin blocks. Blocks containing $5 \times 10^{6} M$. leprae per $\mathrm{ml}$ gelatin $(5 \%, w / v)$ were prepared as described previously (Boddingius \& Dijkman, 1989a).

M. leprae-infected armadillo liver. After dissection from the armadillo, liver segments were stored at $-70^{\circ} \mathrm{C}$ (Royal Tropical Institute, Amsterdam). Segments were kept in liquid nitrogen for transfer to and storage in our laboratory in Rotterdam.

Skin biopsies. Wedge-shaped skin biopsies taken under local anaesthesia from lesions of subpolar or polar lepromatous leprosy patients presenting at the leprosy polyclinic (Department of Dermatology, Academic Hospital Dijkzigt, Erasmus University, Rotterdam), were divided into small segments immediately after excision from the patient. The segments contained epidermis, dermis and subcutaneous tissue. At the time of biopsy, the patients had been treated with dapsone (DDS; $\left.100 \mathrm{mg} \mathrm{d}^{-1}\right)$ and rifampicin $\left(600 \mathrm{mg} \mathrm{d}^{-1}\right)$ for 3 weeks (patient A), or with DDS, rifampicin and clofazimine $\left(50 \mathrm{mg} \mathrm{d}^{-1}\right)$ for 11 months (patient B), or had not yet received anti-leprosy drug treatment (patient $\mathbf{C}$ ).

Fixation procedures. For $M$. leprae-gelatin blocks, the following procedures were used. (a) No fixation; direct immersion in pure cryoprotectant $(2.3 \mathrm{M}$-sucrose in $0.1 \mathrm{M}$-sodium phosphate buffer $\mathrm{pH} 7.3$ ). (b) Fixation for $20 \mathrm{~min}$ in mild fixative added to the cryoprotectant for ultracryotomy $\left[\mathrm{CP}_{2}: 2 \cdot 3 \mathrm{M}\right.$-sucrose containing $2 \%$, $\mathrm{w} / \mathrm{v}$, paraformaldehyde (PF) and $0.25 \%, \mathrm{v} / \mathrm{v}$, glutaraldehyde (GA) in $0.1 \mathrm{M}$-phosphate buffer]. (c) Fixation for $2 \mathrm{~h}$ in mild fixative or very mild fixative consisting of a mixture of $2 \% \mathrm{PF}$ and $0.25 \%$ or $0.1 \% \mathrm{GA}$ in $0.1 \mathrm{M}$-phosphate buffer. This was followed by storage in 2\% PF in $0.1 \mathrm{M}$-phosphate buffer; next, either processing into Lowicryl (see below) or cryoprotection in $\mathrm{CP}_{2}$ (as above) followed by ultracryosectioning was done. (d) Fixation for $2 \mathrm{~h}$ in $3 \%$ GA in $5 \%$ sucrose/phosphate buffer (for preparation, see Boddingius \& Dijkman, 1989a), generally followed by embedding in Lowicryl or by postfixation for $2 \mathrm{~h}$ in $1 \%(\mathrm{w} / \mathrm{v}) \mathrm{OsO}_{4}$, dehydration and embedding in Araldite (see below).

For armadillo liver, mild fixation ( $2 \% \mathrm{PF} / 0 \cdot 25 \% \mathrm{GA}$; procedure $c)$ with omission of storage in $2 \%$ PF was employed, followed by ultracryotomy; alternatively, fixation procedure $d$ (above) and Araldite embedding were utilized.

For human skin biopsy segments, fixation procedure $(d)$ was used, involving $\mathrm{GA} / \mathrm{OsO}_{4}$ fixation and Araldite embedding. In addition, fixation and storage (for 6 years) in 3\% GA in 5\% sucrose/phosphate buffer (fixation procedure $e$ ) was employed followed by Lowicryl embedding.

Lowicryl embedding. After either mild fixation ( $2 \% \mathrm{PF} / 0 \cdot 25 \% \mathrm{GA})$ or very mild fixation ( $2 \% \mathrm{PF} / 0.1 \% \mathrm{GA}$; see $c$ above), after "hard" fixation in GA (see $d$ above), or after fixation and long-term storage in GA (see $e$ above), two differing dehydration and embedding schemes in Lowicryl were employed. In the conventional low-temperature scheme, carried out at -20 or $-30^{\circ} \mathrm{C}$ (Balzer type TTP010 low-temperature polymerization apparatus), a relatively long $(30-60 \mathrm{~min})$ immersion in graded alcohols $(50,80,90,100 \%, v / v$, ethanol $)$ and $1-2 \mathrm{~h}$ immersion in ethanol/Lowicryl $\mathrm{K}_{4} \mathrm{M}$ mixtures were used. This was followed by embedding in pure $\mathbf{K}_{\mathbf{4}} \mathrm{M}$ (Chemische Werke Lowi, Waldkraiburg, FRG). In the short cold-room scheme, designed by us, dehydration and embedding were carried out at cold-room temperature $\left(\sim 4^{\circ} \mathrm{C}\right)$; the steps were the same as described above for the conventional scheme but each step did not exceed $30 \mathrm{~min}$. In the last step (pure $\mathrm{K}_{4} \mathrm{M}$ ) in the coldroom, a Chromato Vue Cabinet (UVP Inc., San Gabriel, USA) was 
employed to photopolymerize $\mathbf{K}_{\mathbf{4}} \mathbf{M}$ by ultraviolet illumination. Lowicryl-embedded tissues were stored at room temperature, tightly sealed with parafilm.

Araldite embedding. Dehydration and embedding procedures of specimens fixed by procedure $d$ (above) have been described previously (Boddingius \& Dijkmañ, 1989a). During dehydration (step 5), overnight alc. $70 \%$ followed alc. $60 \%$.

Ultracryosectioning and ultramicrotomy. The methods are described by Boddingius \& Dijkman (1989a).

Monoclonal antibodies (mAbs). mAb F 47-21-3 specific for $M$. leprae phenolic glycolipid (Kolk et al., 1985), was used.

Gold-labelled secondary antibody. Gold $(10 \mathrm{~nm})$ labelled goat antimouse IgG (GAMG ${ }_{10}$; Janssen Life Sciences Product, Beerse, Belgium) was employed at a dilution of $1: 50$ in 1\% BSA in PBS.

Immunogold labelling of ultrathin sections. During labelling at room temperature, grids with sections were floated upside down on liquid drops placed on wide Parafilm sheets covered with a Perspex cover. For ultracryosections and for Araldite sections, labelling schemes have been described in detail elsewhere (Boddingius \& Dijkman, 1989a); $5 \%(\mathrm{v} / \mathrm{v}) \mathrm{H}_{2} \mathrm{O}_{2}$ was used for the etching of Araldite sections. For Lowicryl sections, swine serum pretreatment was found unnecessary; hence after brief rinsing in PBS, grids were directly incubated for $30 \mathrm{~min}$ in primary antibody (mAb F 47-21-3), diluted $1: 1000$ in PBS. The labelling procedures were otherwise the same as for Araldite sections.

Semi-quantification of gold labelling. To assess the influence of fixation on PGL-I immunogenicity, relative frequencies were determined of gold-labelled 'non-fixed' $M$. leprae and 'fixed' $M$. leprae, using ultracryosections of $\boldsymbol{M}$. leprae-gelatin blocks. Counts of gold-labelled cells were divided by total numbers $(100-400)$ of organisms screened per grid and then multiplied by 100 . Counts were carried out at a magnification of $12000 \times$ (Philips EM 300 electron microscope).

Control labelling experiments. In labelling schemes (see immunogold labelling, above), specific primary antibody (mAb F 47-21-3) either was omitted or was replaced by PBS or by control mAb (F 85-10). mAb F 85-10, directed against a distinct peptidoglycolipid of the MAIS complex (M. avium/M. intracellulare/M. scrofulaceum), was used at a dilution of $1: 1000$ in PBS.

$M$. leprae-specificity testing of $m A b F$ 47-21-3 by immunogold labelling. Ultracryosections of 'non-fixed' $M$. tuberculosis, $M$. nonchromogenicum and $M$. leprae, each species contained in a separate gelatin block, were subjected to identical immunogold labelling procedures (see above). $M$. tuberculosis and $M$. nonchromogenicum suspensions utilized for the preparation of gelatin blocks consisted of heat-killed $\left(10 \mathrm{~min}\right.$ at $\left.80^{\circ} \mathrm{C}\right)$ whole $M$. tuberculosis in PBS and of whole $M$. nonchromogenicum in PBS. Both species had been grown in Sauton's medium.

\section{Results}

PGL-I sites in isolated M. leprae and control experiments with $M$. tuberculosis and $M$. nonchromogenicum

Ultracryosections. Bacterial substructures, distinguishable in GA/O $\mathrm{SSO}_{4}$-fixed and Araldite-embedded (human) $M$. leprae (Fig. 1a), were not clearly imaged in 'nonfixed' ultracryosectioned isolated $M$. leprae. The location of PGL-I given below for ultracryosections is thus approximate.
Capsular material, still surrounding some of the isolated $M$. leprae in a given section, showed gold labelling throughout (see Fig. 5a). PGL-I gold labelling in cell wall areas was pronounced, particularly in dividing bacilli (Fig. 1b). Intracytoplasmic labelling of PGL-I or its antigenic oligosaccharide, although relatively sparse, was a consistent feature in ultracryosectioned $M$. leprae but, because of the electron density of the bacilli, this is not clearly visible in electron micrographs. The gelatin matrix was practically free from gold particles (Fig. 1b).

In control labelling experiments of ultracryosections of 'non-fixed' $M$. tuberculosis and $M$. nonchromogenicum, no gold labelling was seen on $M$. nonchromogenicum (Fig. $2 a$ ) and no gold labelling was present in capsular regions and cell wall areas of $M$. tuberculosis (Fig. 2b). When electron micrographs of 100 immunolabelled $M$. tuberculosis organisms were screened, $7 \%$ of the organisms were found to display an occasional gold particle on the cytoplasm (Fig. 2b). A comparable low level of goldlabelling was seen intrabacterially in $M$. leprae when control mAb F 85-10 was used as primary antibody. The great majority of $M$. leprae incubated with $\mathrm{mAb} F$ 85-10 were free of gold particles (Fig. $2 c$ ). The results indicated the absence of appreciable cross-reactivity of mAb 47 21-3 with either intrabacterial or surface glycolipids probably occurring in $M$. nonchromogenicum, or with peribacterial (surface, capsular) glycolipids in $M$. tuberculosis.

Ultracryosections of isolated $M$. leprae were also used to test semi-quantitatively the possible influence on PGL-I immunogenicity of PF/GA fixation, which is also used in the Lowicryl methods (see below). For M. lepraegelatin blocks subjected to 20 min or $2 \mathrm{~h} \mathrm{PF} / \mathrm{GA}$ fixation (procedure $c$ with $0.25 \% \mathrm{GA}$ ) prior to ultracryosectioning and immunolabelling, relative frequencies of goldlabelled cells were determined (Fig. 3). After 2 h PF/GA fixation, satisfactory numbers of gold-labelled $M$. leprae organisms were still present (Fig. 3). Comparable $2 \mathrm{~h}$ PF/GA fixation of infected armadillo liver segments, followed by ultracryosectioning, resulted in distinct PGL-I gold-labelling both on $M$. leprae and outside the bacilli. Such extrabacterial PGL-I was found in the electron-transparent substance in which bacilli were 'floating' in vacuoles of armadillo liver parenchymal cells (illustrated in Boddingius \& Dijkman, 1989b). This showed that PF/GA fixation of $M$. leprae-infected tissues allows satisfactory demonstration of PGL-I both intraand extrabacterially.

Araldite sections of isolated $M$. leprae and of infected armadillo liver. Immunogold labelling experiments showed that after 'hard' fixation in $\mathrm{GA} / \mathrm{OsO}_{4}$ and Araldite embedding, PGL-I immunogenicity in infected 


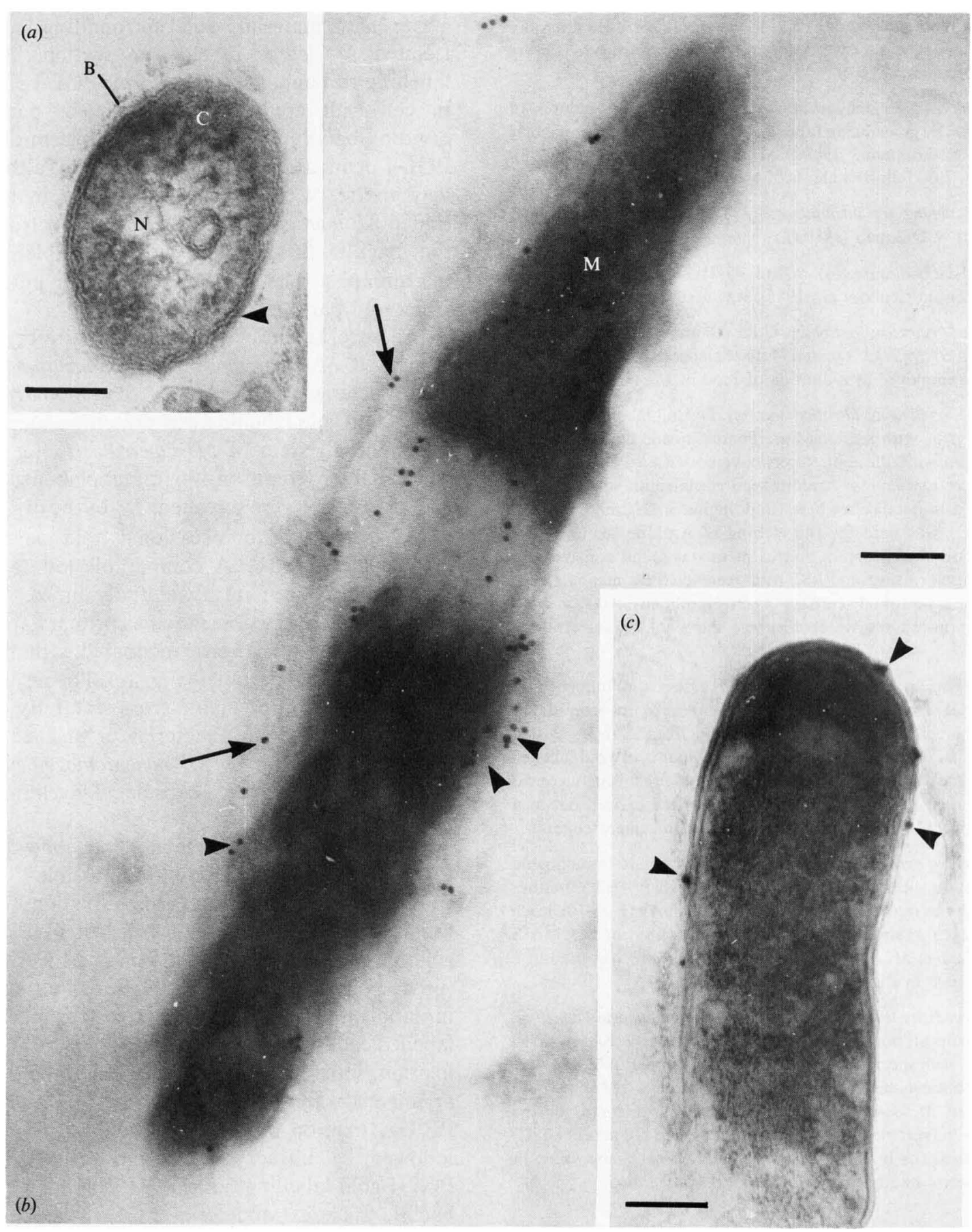

Fig. 1. ( $a, c)$ Araldite sections of 'hard'-fixed human M. leprae either non-labelled (a) or after immunogold labelling of PGL-I (c). (b) Ultracryosection of 'non-fixed' $M$. leprae isolated from armadillo liver; immunogold labelling of PGL-I. Bars, $100 \mathrm{~nm}$. (a) In the intact, transversely sectioned $M$. leprae cell are visible : nucleoplasm $(\mathrm{N})$, cytoplasm $(\mathrm{C})$, double cell membrane $(\rightarrow)$, and peripherally to the membrane the electron-dense part of the cell wall and the cell wall boundary (B). Skin biopsy, lepromatous patient (patient A). (b) Isolated M. leprae $(\mathrm{M})$ caught in the process of division and displaying gold labelling of PGL-I in the cell wall $(\rightarrow)$, in capsular areas $(\rightarrow)$ and, occasionally, in the bacterial cytoplasm. (c) Gold labelling of PGL-I $(-)$ at several sites in the wall of the longitudinally sectioned $M$. leprae cell. Dermal macrophage; same skin biopsy as in (a).

tissues is retained to an acceptable degree (see Boddingius \& Dijkman, 1989b). This is less so for cell wall PGL$\mathrm{I}$ in isolated $\boldsymbol{M}$. leprae, while $\boldsymbol{M}$. leprae capsules are not detectable (see Boddingius \& Dijkman, 1989a).
Lowicryl sections. After very mild fixation (procedure $c$ with $0.1 \% \mathrm{GA}$ ) and PF storage of isolated $M$. leprae in gelatin, followed by embedding in Lowicryl (using the long low-temperature scheme or the shorter cold-room 

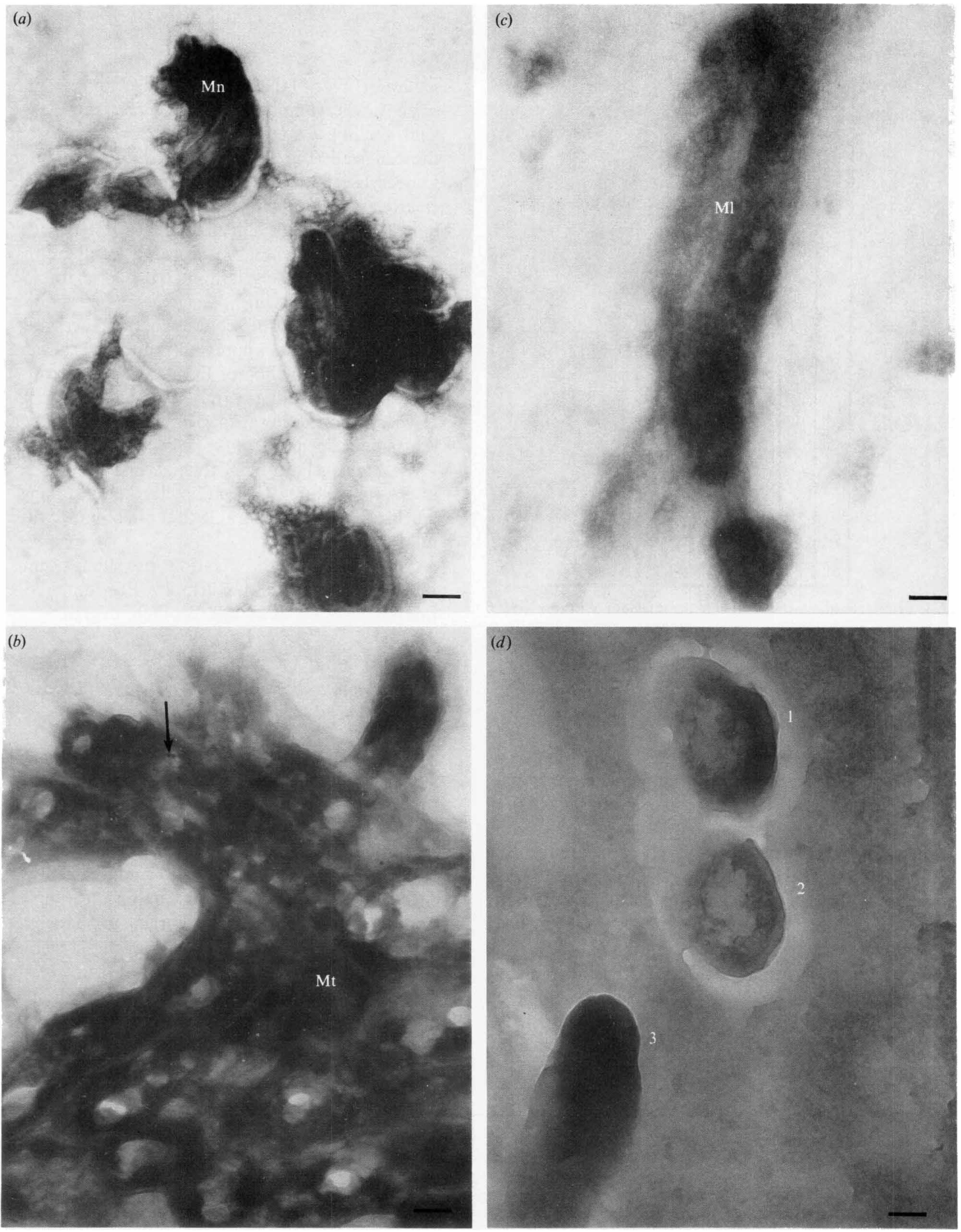

Fig. 2. Control gold labelling of $M$. nonchromogenicum and $M$. tuberculosis with mAb F 47-21-3 $(a, b)$ and of $M$. leprae with $\mathrm{mAb} F$ 85-10 $(c, d)$ and with $\mathrm{GAMG}_{10}$. Gold labelling is absent on the mycobacterial organisms $(a, c, d)$ and in the macrophage cytoplasm $(d)$. Bars, $100 \mathrm{~nm}$. (a) Ultracryosection of 'non-fixed' $M$. nonchromogenicum organisms (Mn) dispersed in gelatin. (b) Ultracryosection of 'nonfixed' $M$. tuberculosis organisms (Mt) dispersed in gelatin and occurring in clusters. Gold labelling is absent on cell walls and capsular areas, but a sporadic gold particle is seen on the bacterial cytoplasm of a few organisms $(\rightarrow)$. (c) Ultracryosection of 'non-fixed' isolated $M$. leprae (Ml) in gelatin. (d) Lowicryl section with transversely cut $(1,2)$ and obliquely sectioned $(3) M$. leprae in a dermal host macrophage in a human lepromatous skin lesion (patient $\mathrm{C}$ ). Lowicryl cold room embedding. 


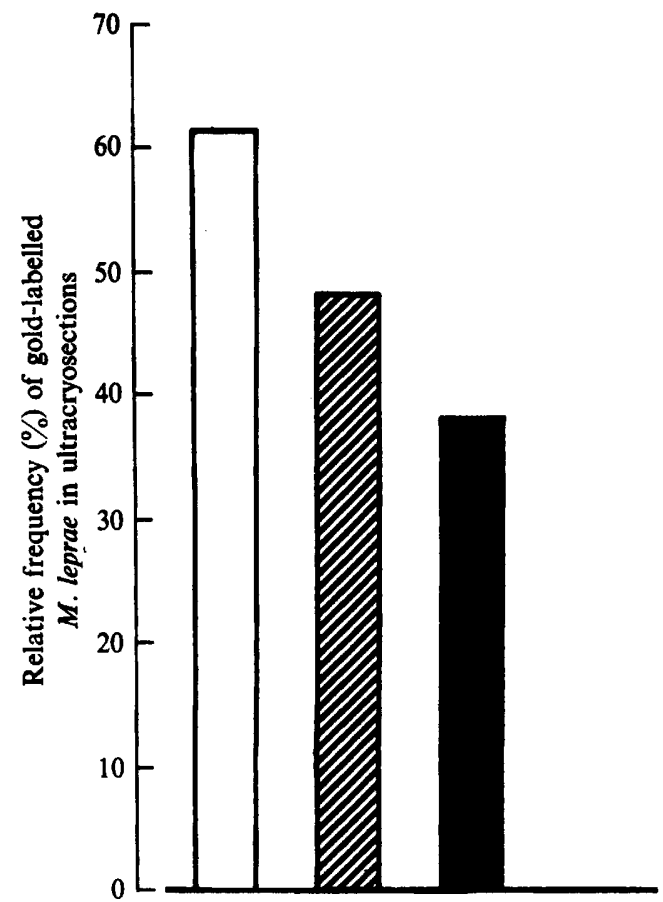

Fig. 3. Semi-quantitative evaluation of the influence of fixation on the

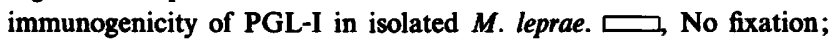
$\mathrm{m}$, mild fixation for $20 \mathrm{~min}$ in $\mathrm{CP}_{2}$ during cryoprotection; mild PF/GA fixation ( $2 \% \mathrm{PF} / 0.25 \% \mathrm{GA}$ ) followed by $20 \mathrm{~min}$ in $\mathrm{CP}_{2}$ during cryoprotection.

scheme) the following was observed. Peri- and intrabacterial PGL-I gold-labelling intensities were higher than seen in ultracryosections and Araldite sections, while labelled bacterial structures largely appeared to be identical. After Lowicryl-immunogold labelling procedures, dividing $M$. leprae organisms displayed many more PGL-I sites in the cell wall (Fig. $4 a$ ) than were discernible in ultracryosectioned dividing $M$. leprae (Fig. $1 b$ ). Also the transverse cell walls of the incompletely separated daughter cells showed gold labelling in Lowicryl preparations and the cytoplasm was labelled in several places (Fig. 4a). In addition, peri-bacterial (capsular) PGL-I was more clearly localized in Lowicryl sections (Fig. 5b) than in ultracryosections (Fig. 5a). In some Lowicryl-embedded $M$. leprae, capsular PGL-I gold labelling was confined to a narrow zone distant from the cell wall (Fig. 5b). Another finding of interest in Lowicryl sections was that numerous gold particles, not clearly associated with bacterial structures, were found quite evenly dispersed in all of the 'vacuolar spaces' (Fig. $4 c$ ) in which individual $M$. leprae or small groups of $M$. leprae resided in the gelatin matrix. Comparable vacuolar spaces surrounding $M$. leprae in ultracryosections (Fig. $1 b$ ) or in Araldite sections contained very few or no gold particles. No appreciable differences were seen between gold labelling of PGL-I after low-temperature Lowicryl embedding (Fig. 4a) and after cold-room Lowicryl embedding (Fig. $5 b$ ) of $M$. leprae taken from the same suspension.

$M$. leprae-gelatin blocks which had been 'hard'-fixed in GA (procedure $d$ ) before Lowicryl embedding showed labelling results comparable to those obtained after very mild fixation.

Thus none of the fixation procedures employed, including storage for $1-7 \mathrm{~d}$ in $2 \% \mathrm{PF}$, impaired PGL-I immunogenicity to an unacceptable degree.

\section{Intra- and extrabacterial PGL-I in human leprosy skin biopsies embedded in Lowicryl or Araldite}

Araldite sections. In sections of 'hard' $\left(\mathrm{GA} / \mathrm{OsO} \mathrm{O}_{4}\right)$-fixed and Araldite-embedded human skin (patient $\mathrm{A}$; fixation procedure $d$ ) PGL-I gold labelling was seen, in dermal macrophages containing $M$. leprae, in the following sites: (1) on cell walls of electron-dense bacilli representing viable $M$. leprae (Figs $1 c, 5 c$ ) and on cell walls of degenerating $M$. leprae (Fig. $5 c, d$ ), (2) on debris, possibly of bacterial origin, occurring in phagolysosomal embedded isolated $M$. leprae, however, showed a more precise immuno-localization of PGL-I (Fig. $5 b$ ), suggesting that capsular PGL-I mainly occurs in a confined zone about $85 \mathrm{~nm}$ from the cell wall. This 'PGL-I zone' seems to agree with the position suggested for phenolphthiocerol-based mycosides in mycobacterial envelopes

Fig. 4. Immunogold labelling of Lowicryl sections with $\mathrm{mAb} \mathrm{F}$ 47-21-3 and $\mathrm{GAMG}_{10}$. (a) Isolated $M$. leprae caught in the process of division. Note many gold labelled PGL-I sites in the cell wall $(\rightarrow)$, including the new transverse cell walls $(\Rightarrow)$ which separate the daughter cells. In several places the bacterial cytoplasm contains gold-labelled sites $(\boldsymbol{D})$ which represent either PGL-I sites or locations of its antigenic oligosaccharide. Fixation procedure $c(2 \%$ PF/ $0.1 \%$ GA); low-temperature Lowicryl embedding. Bar, $50 \mathrm{~nm}$. (b) Electron-dense $M$. leprae residing in human dermal macrophage in skin lesion (patient C). Note PGL-I labelling in cell wall areas of $M$. leprae $(\rightarrow)$ and, outside the bacillus, in the electron-transparent substance (ETS) of the phagolysosomal vacuole. Fixation (6 years) in $3 \%$ GA. Lowicryl cold-room embedding. Bar, $100 \mathrm{~nm}$. (c) Heavy gold labelling $\rightarrow$ of (?)soluble PGL-I in vacuoles which contain isolated $M$. leprae $(M)$. Cell walls $(>)$ and cytoplasm of $M$. leprae are also labelled. The gelatin matrix $(G)$ is practically free from gold particles. Fixation procedure $c(2 \% \mathrm{PF} / 0 \cdot 1 \% \mathrm{GA})$. Lowicryl cold-room embedding. Bar, $100 \mathrm{~nm}$. (d) Extrabacterial PGL-I $\rightarrow$ ) occurring in the electron-translucent substance (ETS) in a phagolysosomal vacuole also containing $M$. leprae (M) with gold-labelled PGL-I sites. Note that PGL-I is not expressed on the exterior of the macrophage's cell membrane ( $>$ ). The extracellular space (ES) does not show PGL-I labelling. Fixation (6 years) in 3\% GA. Lowicryl cold-room embedding. Bar, $100 \mathrm{~nm}$. 

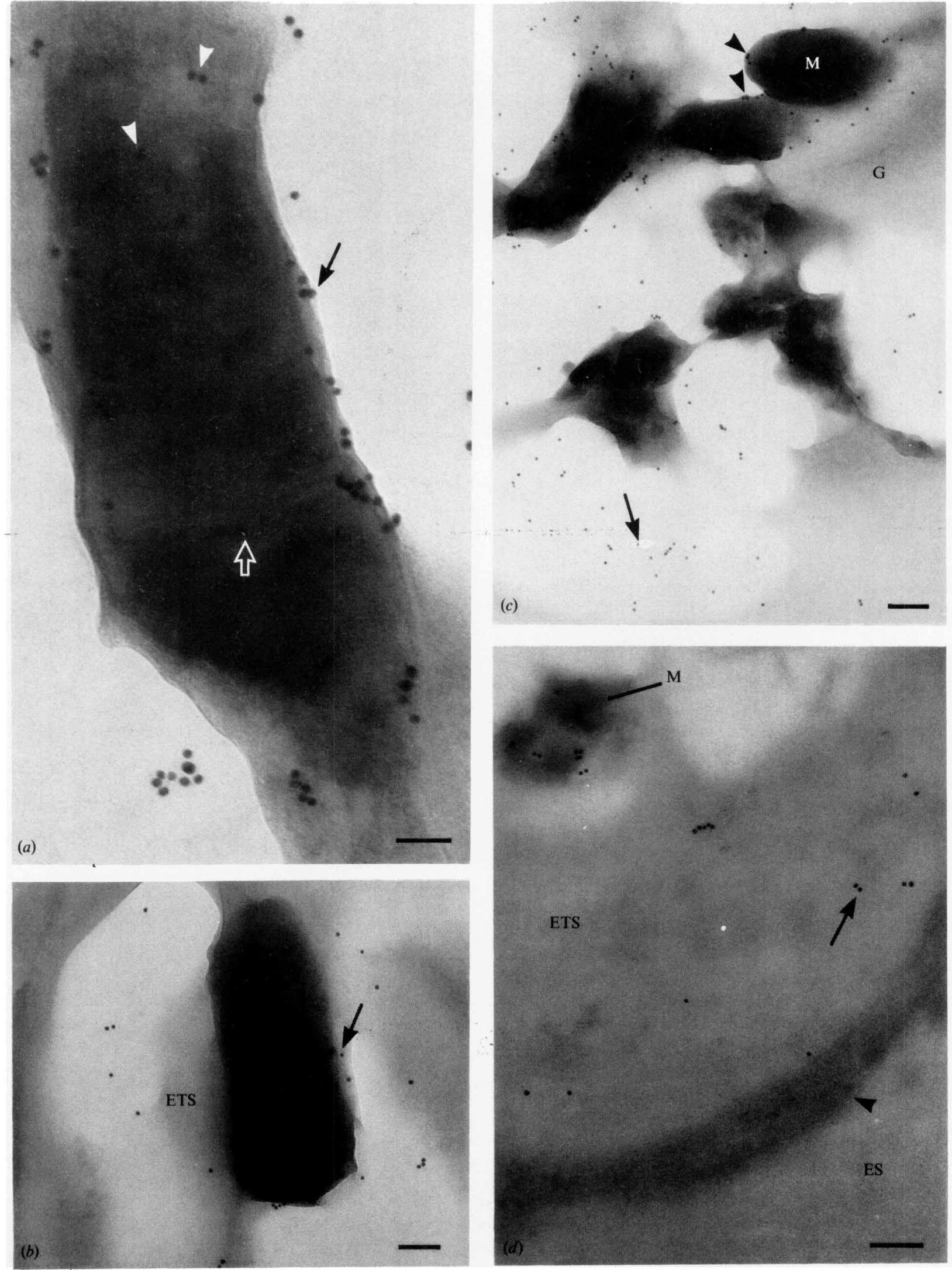

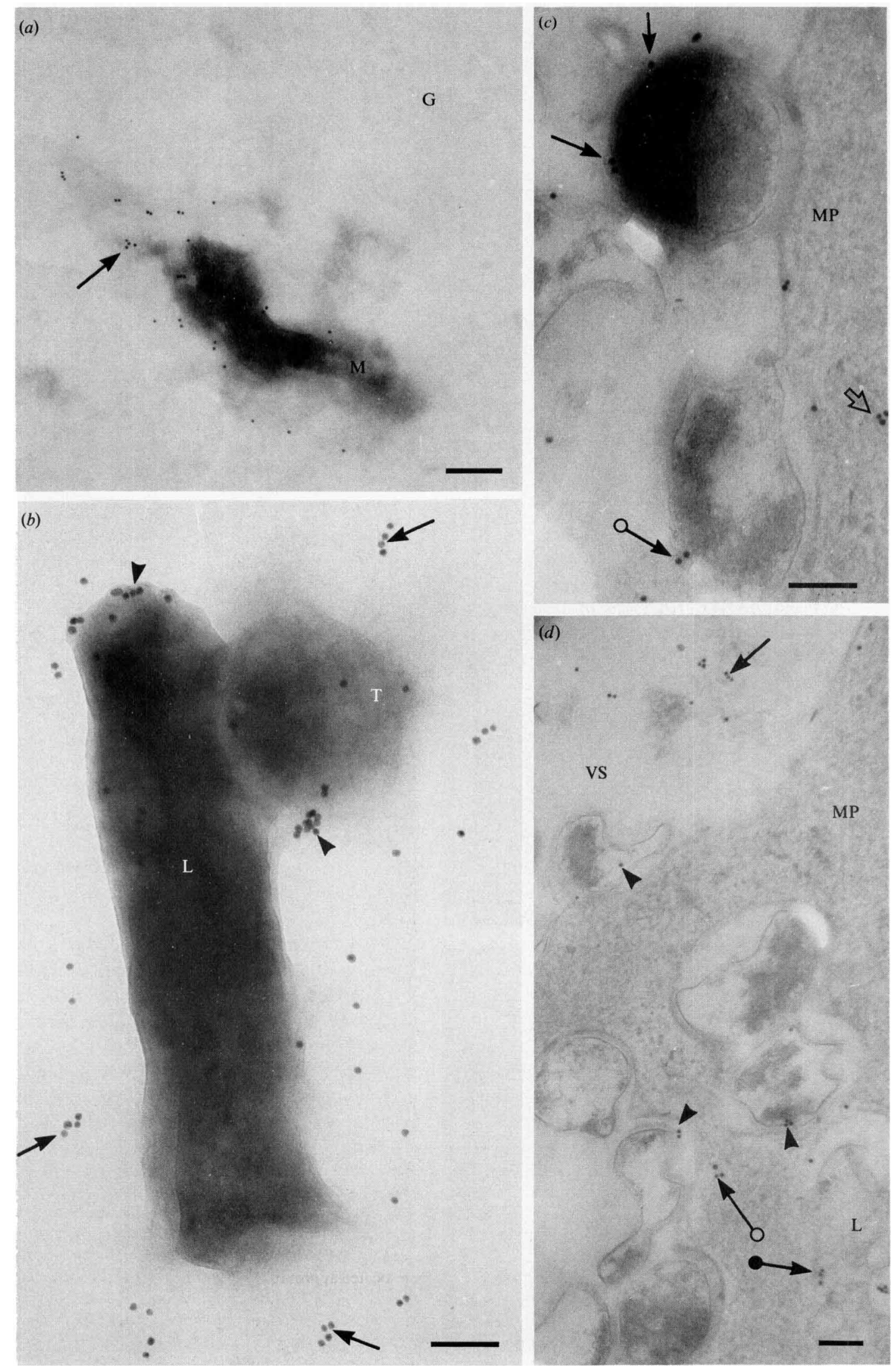
large vacuoles (Fig. $5 d$ ), (3) on the boundary between phagolysosomal vacuoles and the macrophage cytoplasm proper (Fig. 5c), and (4) in small vacuoles of $M$. lepraecontaining lysosomes, which have a finely foamy appearance (Fig. $5 d$ ). The cytoplasm proper of dermal macrophages only occasionally showed gold labelling. In the skin biopsy of patient B (treated for nearly 1 year with anti-leprosy drugs), PGL-I labelling was seen in a few places only, notably in large phagolyosomal vacuoles containing bacterial debris in 'foamy' dermal macrophages.

Lowicryl sections. Prior to Lowicryl embedding, skin biopsy segments had been 'hard'-fixed in GA followed by storage for 6 years in this fixative (fixation procedure $e$ ). The first contact with GA-fixative had been within seconds of excision of the biopsy from the patient.

In dermal macrophages, intrabacterial PGL-I gold labelling was seen in the cytoplasm and cell wall of $\boldsymbol{M}$. leprae and just outside the bacillus, possibly in the former capsular area (Fig. $4 b$ ). Approximately $20 \%$ of the $M$. leprae organisms in a given skin section showed gold labelling. Extrabacterial PGL-I gold labelling was seen in two major positions in the dermal host macrophage: (1) in the electron-translucent substance surrounding the 'PGL-I positive' bacilli in phagolysosomal vacuoles (Fig. $4 b, d$ ), and (2) in the cytoplasmic stroma of the macrophage. PGL-I gold labelling was not a consistent feature in the electron-translucent substance of all the $M$. leprae-containing vacuoles: in the same 'foamy' macrophage, some $M$. leprae-containing vacuoles were abundantly labelled, other vacuoles not at all. The outer surface of the cell membrane of dermal macrophages containing $M$. leprae and extrabacterial PGL-I showed no PGL-I gold-labelled sites (Fig. 4d). Certain other cells, containing many $M$. leprae bacilli, and surrounded by a basal lamina, showed no PGL-I gold labelling. These cells were possibly Schwann cells of tiny dermal nerves, as such cells were readily observed in nonlabelled Araldite sections of the same biopsy.

In the epidermis, extrabacterial PGL-I gold labelling was found in the cytoplasm of $M$. leprae-containing epidermal basal cells and in extracellular spaces between such basal cells. The skin segments used were too narrow to contain adequate numbers of Langerhans' cells for the possible involvement of these cells in PGL-I expression to be evaluated.

No significant differences in amounts of gold labelling of PGL-I were seen when we compared results obtained after conventional low-temperature Lowicryl embedding and after cold-room embedding of segments from the same biopsy. Some reduction in the intensity of the electron beam was necessary during examination of Lowicryl sections of cold-room embedded specimens in order to avoid disruption of the electron-translucent substance in $M$. leprae-containing macrophages.

Ultrastructural details of bacilli and of host cells, in both types of Lowicryl sections, were not preserved to such a satisfactory degree as in skin segments which had been post-fixed with $\mathrm{OsO}_{4}$ and embedded in Araldite.

\section{Control labelling of Lowicryl and Araldite sections}

When mAb F 47-21-3 was omitted or was replaced by PBS or by control mAb F 85-10 (Fig. 2d), which is not directed against PGL (see Methods), no gold labelling was observed in any of the sections.

\section{Discussion}

In previous studies on PGLs, the localization of PGL-I in $M$. leprae and in $M$. leprae-infected tissue has been rather superficial. As PGL-I was easily separable mechanically from $M$. leprae, the antigen was thought to occur in the bacterial capsule, and this was supported by immunofluorescent staining of whole $M$. leprae (Young et al., 1984).

Our Lowicryl-immunogold procedures presented here, and immunolabelling results obtained on ultracryosections, give semi-quantitative results and show convincingly that PGL-I occurs in the bacterial capsular area. In ultracryosections, ragged material thought to represent remnants of capsular material showed randomly distributed gold labelling (Fig. 5a). Some of the Lowicryl-

Fig. 5. Comparison between immunogold labelling of PGL-I in 'non-fixed' ultracryosectioned isolated $M$. leprae (a), in very mildly fixed and PF-stored Lowicryl- (cold-room) embedded isolated $M$. leprae (b), and in M. leprae residing in 'hard'-fixed and Aralditeembedded human skin, also showing extrabacterial PGL-I $(c, d)$. (a) PGL-I labelling $(\rightarrow)$ is seen throughout the bacterial capsular material of isolated $M$. leprae $(\mathrm{M})$. Note that the gelatin matrix $(G)$ is free from labelling. Bar, $140 \mathrm{~nm}$. (b) Longitudinally sectioned isolated $M$. leprae $(\mathrm{L})$ and transversely cut organism $(\mathrm{T})$ showing gold labelling on cell wall areas $(\longrightarrow)$ and on capsular areas $(\rightarrow)$. Note that most of the gold particles in capsular areas of $L$ are confined to a zone located approximately $85 \mathrm{~nm}$ from the cell wall. Bar, $100 \mathrm{~nm}$. (c) Human dermal host macrophage displaying gold particles on the cell wall $\rightarrow$ of electron-dense $M$. leprae and on the cell wall of a degenerating $M$. leprae $(\mathrm{O} \rightarrow$ ), at the boundary between lysosome and macrophage cytoplasm and in the macrophage cytoplasm (MP) $(\Leftrightarrow)$. Bar, $100 \mathrm{~nm}$. (d) Extrabacterial PGL-I $(\rightarrow)$ is present on (?)bacterial debris in vacuolar space (VS) also containing $M$. leprae. Cell walls show PGL-I labelling $(-)$ in degenerating $M$. leprae, and PGL-I is also labelled $(\longrightarrow)$ in the finely vacuolar lysosome (L). Sporadic labelling is seen on the macrophage cytoplasm $(\mathrm{MP} ; \mathrm{O} \rightarrow$ ). Bar, $100 \mathrm{~nm}$. 
on the basis of the likely location of envelope components (Minnikin, 1982; Brennan, 1988). In these models of the mycobacterial envelope, phenol-phthiocerol-based mycosides are located in a hydrophobic interaction region, in the periphery of the envelope, with their hydrophilic oligosaccharide moieties projecting outside this region.

In addition, we have localized PGL-I (or its antigenic oligosaccharide) in other substructures of $M$. leprae, predominantly in the cell wall and, to a lesser extent, in the bacterial cytoplasm. The gold-labelled sites seen in the $M$. leprae cytoplasm could in part represent sites of synthesis of specific oligosaccharide; they deserve further study. The very sporadic labelling in the cytoplasm that was observed in about $7 \%$ of the $M$. tuberculosis organisms screened may represent specific labelling, as it was not observed in M. nonchromogenicum. If so, this would seem to suggest a slight cross-reactivity of mAb F 47-21-3 with intracytoplasmic compounds in $M$. tuberculosis. The significance of this observation remains to be elucidated. There was no cross-reactivity of $\mathrm{mAb} F$ 47-21-3 with capsular (envelope) compounds of $M$. tuberculosis and $M$. nonchromogenicum, thus supporting the finding of $M$. leprae specificity of $\mathrm{mAb} F$ 47-21-3 in immunofluorescence tests on whole mycobacteria (Kolk et al., 1985).

Hydrophilic Lowicryl $\left(\mathrm{K}_{\mathbf{4}} \mathbf{M}\right)$ resin, used here as an embedding medium for immunodetection of PGL-I, offers reasonably good resolution for transmission electron microscopy of, amongst others, bacteria and mitochondria (Armbruster et al., 1982). $\mathrm{K}_{4} \mathrm{M}$ resin has not previously been used for the immunocytochemical study of glycolipids; it is generally used for immunodemonstration of proteins (e.g.. enzymes) in tissues (Roth et al., 1981; Roth \& Berger, 1982; Kellenberger et al., 1987). One important finding we made is that Lowicryl sections give more satisfactory quantitative immunogold labelling of PGL-I than do ultracryosections or Araldite sections. Secondly, it was shown that neither (very) mild nor 'hard' fixation, even 'hard' fixation of very long (6 years) duration, influenced PGLI immunogenicity to an unacceptable degree. This discovery offers numerous possibilities for the future use (for the immunocytological or histological demonstration of PGL-I) of a wide range of leprosy tissues stored in various fixatives. Izumi et al. (1985) previously showed PGL-I seropositivity in infected human lepromatous liver kept for a long time in formalin.

The PGL-I sites shown here in Lowicryl sections compared well with, and were present in larger amounts than, those observed in ultracryosections and Araldite sections of isolated $M$. leprae. An abundance of PGL-I sites was seen in the cell wall of many isolated $M$. leprae, particularly in dividing organisms, in which new cell wall and capsular material must be synthesized. Dividing $M$. leprae organisms were encountered very infrequently: not more than one pair of longitudinally sectioned dividing $M$. leprae was seen in all sections examined of a given $M$. leprae-gelatin block. This prevented a more quantitative evaluation of PGL-I in dividing organisms. Precise quantification of PGL-I immunogold labelling was not attempted in our study. However, the heavy labelling we obtained in individual isolated $M$. leprae by the Lowicryl-immunogold method is compatible with the biochemical report by Cho et al. (1986) that $2 \%$ of the mass of $M$. leprae is composed of PGL-I.

The subcellular location of PGL-I in M. leprae located in situ in human skin lesions agreed well with that seen on isolated M. leprae. However, while most of the isolated $M$. leprae examined in a given Lowicryl section showed gold labelling, only some (at most $20 \%$ ) of the $M$. leprae residing in skin segments of an untreated multibacillary patient showed gold-labelled sites in Lowicryl sections. In view of the large amounts of solid (viable) M. leprae occurring in these skin segments this was an unexpected finding. Possible explanations are that mAb F 47-21-3, directed against PGL-I from isolated $M$. leprae, reacts best with PGL-I in isolated $M$. leprae, and less well with human $M$. leprae, or that the long (6 years) duration of 'hard' GA fixation to which the human $M$. leprae had been subjected reduced the immunogenicity of PGL-I. However, the presence of heavily labelled $M$. leprae (Fig. $4 b$ ) next to non-labelled $M$. leprae organisms in Lowicryl sections of human skin, seems to disallow the second possibility and to weaken the first. A third possibility, which we consider the most likely explanation, is that the $M$. leprae organisms isolated from armadillo liver were all in a biochemically active state in which ample PGL-I is present in the cell wall and capsule, while many of the $M$. leprae residing in macrophages in human skin were in a biochemically less active state. The possible effect of the nature of the host tissue on PGL-I quantities should be investigated further. This could be done by comparing labelling results obtained with freshly excised or liquidnitrogen-stored segments from infected armadillo liver with those obtained with freshly excised segments of skin lesions (and, where possible, with autopsied or formaldehyde-stored liver) from untreated multibacillary patients.

The present study does not differentiate between the possible occurrence of soluble PGL-I antigen and membrane- (or otherwise structurally-) bound PGL-I. Our observations, in Lowicryl sections, of abundant extrabacterial PGL-I in all the vacuoles containing isolated $M$. leprae within gelatin blocks suggest that our Lowicryl embedding procedures may have prevented soluble PGL-I from diffusing out of $M$. leprae-gelatin blocks into lipid-solvents employed in dehydration. 
Whether the PGL-I sites demonstrated in Lowicryl sections in the human dermal macrophage cytoplasm proper likewise present soluble PGL-I remains to be elucidated.

Our observations of extrabacterial PGL-I are in agreement with earlier conclusions that PGL-I must be present extrabacterially in $M$. leprae-infected armadillo liver (Hunter \& Brennan, 1983) and in skin from humans infected with leprosy (Young, 1981), since the biochemically shown quantities of PGL-I or of bacterial lipid were far in excess of that expected from the bacillary load.

Sites of extrabacterial PGL-I occurrence in human skin were identified in Lowicryl-embedded specimens, both in a very satisfactory quantitative manner and in a wider distribution than seen in the qualitatively superior Araldite sections. Extrabacterial PGL-I was found in the basal epidermal cells containing $M$. leprae and just outside these cells in an untreated multibacillary patient. The many $M$. leprae-containing dermal macrophages encountered failed to show gold labelling at the outside of the macrophage cell membrane (Fig. $4 d$ ). This suggests that in the skin areas of multibacillary patients studied here, PGL-I was not expressed by macrophages. In this context, the low amount of HLA-DR antigen expressed by inflammatory cells in hyperbacillary forms of leprosy (Collings et al., 1985) is interesting. The possible association between these phenomena should be investigated by double immunolabelling to identify both antigens simultaneously. The varying degree of extrabacterial PGL-I gold labelling seen, in Lowicryl sections, in $M$. leprae-containing vacuoles of the same dermal macrophage may reflect differences, in either metabolism or growth, between individual clusters of $M$. leprae.

The drawback of the Lowicryl method in immunolabelling studies of tissues is the poorer preservation of ultrastructural details. To complement each other in PGL-I localization studies, Lowicryl and Araldite embedding should preferably be carried out concomitantly for segments of the same skin biopsy or leproma.

In a preliminary test, we were unable to demonstrate satisfactorily the $M$. leprae-specific $36 \mathrm{kDa}$ and the $65 \mathrm{kDa}$ protein antigens in very mildly fixed and lowtemperature Lowicryl-embedded isolated $M$. leprae. A combination of storage of isolated $M$. leprae in the deepfreeze, subsequent thawing, and ensuing fixation may be responsible for these negative findings. The only technique thus far found suitable for both these protein antigens has been ultracryosectioning of 'non-fixed' specimens (Boddingius \& Dijkman, 1989b). We recommend that for future simultaneous demonstration of PGL-I and protein antigens in any study on possible differences in the expression of lipid and protein antigens by macrophages or Langerhans' cells, freshly excised and 'non-fixed' $M$. leprae or $M$. leprae-infected tissue segments are sectioned by advanced ultracryotomy. Next, double-immunogold labelling should be done as described by Geuze et al. (1981).

In conclusion, the results presented here show the suitability of Lowicryl embedding for the quantitative demonstration of intra- peri- and extrabacterial PGL-I antigen sites in skin from human leprosy cases and in $\boldsymbol{M}$. leprae isolated from armadillo liver. Araldite embedding gave qualititatively the best immunogold labelling results. The cold-room Lowicryl embedding variant, employing a cost-effective apparatus, proved to be highly efficient for demonstration of PGL-I. Similar methods to those established here for one important lipid, PGL-I, deserve to be tested for another immunodominant glycolipid of $M$. leprae, namely lipo-arabinomannan (LAM-B; Hunter et al., 1986), which has to date been the subject of only a single light-microscopic investigation (Khanolkar et al., 1989).

Financial support from the Netherlands Leprosy Relief Organization (NSL) and from the Gastmann Wichers Foundation enabled the work presented here to be carried out. Monoclonal antibodies (F 47-213; F 85-10), isolated $M$. leprae and other mycobacterial suspensions were kindly provided by $\mathrm{Dr}$ A. Kolk and collaborators.

Dr M. J. Pearson, Amsterdam, is thanked for reading and correcting the English text.

\section{References}

Agis, F., Schlich, P., Cartel, J. L., Guidi, C. \& Bach, M.-A. (1988). Use of anti-M. leprae phenolic glycolipid-I antibody detection for early diagnosis and prognosis of leprosy. International Journal of Leprosy 56, 527-536.

Armbruster, B. L., Carlemalm, E., Chiovetti, R., Garavito, R. M. Hobot, J. A., Kellenberger, E. \& Villiger, W. (1982). Specimen preparation for electron microscopy using low temperature embedding resins. Journal of Microscopy 126, 77-85.

BARKSDALE, L. \& KIM, K.-S. (1977). Mycobacterium. Bacteriological Reviews 41, 217-372.

BodDingIUs, J. (1974). The occurrence of Mycobacterium leprae within axons of peripheral nerves. Acta Neuropathologica 27, 257-270.

BoDDINGIUS, J. (1976). Ultrastructural changes in blood vessels of peripheral nerves in leprosy neuropathy. I. Tuberculoid and borderline-tuberculoid leprosy patients. Acta Neuropathologica 35, $159-181$.

BoDDINGIUS, J. (1977). Ultrastructural changes in blood vessels of peripheral nerves in leprosy neuropathy. II. Borderline, borderlinelepromatous and lepromatous leprosy patients. Acta Neuropathologica 40, 21-39.

BoDDINGIUS, J. (1984). Ultrastructural and histophysiological studies on the blood-nerve barrier and perineurial barrier in leprosy neuropathy. Acta Neuropathologica 64, 282-296.

Boddingius, J. \& DiJkman, H. P. (1989a). Immunogold labeling method for Mycobacterium leprae-specific phenolic glycolipid in glutaraldehyde-osmium fixed and Araldite-embedded leprosy lesions. Journal of Histochemistry and Cytochemistry 37, 455-462.

Boddingius, J. \& DiJkman, H. $(1989 \mathrm{~b})$. In situ locations of Mycobacterium leprae-specific antigens. Immuno-electronoptical studies. Acta Leprologica 7 (supplement 1), 107-112. 
Boddingius, J., Dijkman, H. B. P. M., Hendriksen, E. G., Prens, E. P., Muntendam, J. \& Lombardi, C. (1989). Bacillary and phagolysosomal changes in skin lesions of multidrug-treated borderline or lepromatous leprosy patients. Ultramicroscopy 27, 196-197.

BrenNAN, P. J. (1988). Mycobacterium and other actinomycetes. In Microbial Lipids, vol. 1, pp. 203-298. Edited by C. Ratledge \& S. G. Wilkinson. London: Academic Press.

Chanteau, S., Cartel, J.-L., Celerier, P., Plichart, R., Desforges, S. \& Roux, J. (1989). PGL-I antigen and antibody detection in leprosy patients: evolution under chemotherapy. International Journal of Leprosy 57, 735-743.

Cho, S.-N., Yanagihara, D. L., Hunter, S. W., Gelber, R. H. \& BRENNAN, P. J. (1983). Serological specificity of phenolic glycolipid I from Mycobacterium leprae and use in serodiagnosis of leprosy. Infection and Immunity 41, 1077-1083.

Cho, S.-N., Hunter, S. W., Gelber, R. H., Rea, T. H. \& Brennan, P. J. (1986). Quantitation of the phenolic glycolipid of Mycobacterium leprae and relevance to glycolipid antigenemia in leprosy. Journal of Infectious Diseases 153, 560-569.

Collings, L. A., Tidman, N. \& Poulter, L. W. (1985). Quantitation of HLA-DR expression by cells involved in the skin lesions of tuberculoid and lepromatous leprosy. Clinical and Experimental Immunology 61, 58-66.

Fujiwara, T., Hunter, S. W., Cho, S.-N., Aspinall, G. O \& Brennan, P. J. (1984). Chemical synthesis and serology of disaccharides and trisaccharides of phenolic glycolipid antigens from the leprosy bacillus and preparation of a disaccharide protein conjugate for serodiagnosis of leprosy. Infection and Immunity 43, 245-252.

Geuze, H. J., Slot, J. W., Van Der Ley, P. A. \& Scheffer, R. C. T. (1981). Use of colloidal gold particles in double-labeling immunoelectron microscopy of ultrathin frozen tissue sections. Journal of Cell Biology 89, 653-665.

HunTER, S. W. \& BRENNAN, P. J. (1981). A novel phenolic glycolipid from Mycobacterium leprae possibly involved in immunogenicity and pathogenicity. Journal of Bacteriology 147, 728-735.

HUNTER, S. W. \& BRENNAN, P. J. (1983). Further specific extracellular phenolic glycolipid antigens and a related diacylphthiocerol from Mycobacterium leprae. Journal of Biological Chemistry 258, 75567560.

Hunter, S. W., Futiwara, T. \& BrenNan, P. J. (1982). Structure and antigenicity of the major specific glycolipid antigen of Mycobacterium leprae. Journal of Biological Chemistry 257, 15072-15078.

Hunter, S. W., GaYloRd, H. \& BRENNAN, P. J. (1986). Structure and antigenicity of the phosphorylated lipopolysaccharide antigens from the leprosy and tubercle bacilli. Journal of Biological Chemistry 261, $12345-12351$
Izumi, S., Sugiyama, K., Fujiwara, T., Hunter, S. W. \& Brennan, P. J. (1985). Isolation of the Mycobacterium leprae-specific glycolipid antigen, phenolic glycolipid-I, from formalin-fixed human lepromatous liver. Journal of Clinical Microbiology 22, 680-682.

Kellenberger, E., DÜrRenberger, M., Villiger, W., CARLemalm, E. \& WURTZ, M. (1987). The efficiency of immunolabel on Lowicryl sections compared to theoretical predictions. Journal of Histochemistry and Cytochemistry 35, 959-969.

Khanolkar, S. R., Mackenzie, C. D., Lucas, S. B., Hussen, A., GirdhaR, B. K., Katoch, K. \& MCAdaM, K. P. W. J. (1989). Identification of Mycobacterium leprae antigens in tissues of leprosy patients using monoclonal antibodies. International Journal of Leprosy 57, 652-658.

Kolk, A. H. J., Ho, M. L., Klatser, P. R., Eggelte, T. A. \& Portaels, F. (1985). Production of monoclonal antibodies against Mycobacterium leprae and armadillo-derived mycobacteria. Annales de l'Institut Pasteur/Microbiologie 136B, 217-224.

MinnikIN, D. E. (1982). Lipids: complex lipids, their chemistry, biosynthesis and roles. In The Biology of the Mycobacteria, vol. 1, Physiology, Identification and Classification, pp. 95-184. Edited by C. Ratledge \& J. Stanford. London: Academic Press.

Neill, M. A. \& KLEBanofF, S. J. (1988). The effect of phenolic glycolipid-I from Mycobacterium leprae on the antimicrobial activity of human macrophages. Journal of Experimental Medicine 167, 30-42.

ROTH, J. \& BERGER, E. G. (1982). Immunocytochemical localization of galactosyltransferase in HeLa cells. codistribution with thiamine pyrophosphatase in trans-Golgi cisternae. Journal of Cell Biology 93, 223-229.

Roth, J., Bendayan, M., Carlemalm, E., Villiger, W. \& Garavito, M. (1981). Enhancement of structural preservation and immunocytochemical staining in low temperature embedded pancreatic tissue. Journal of Histochemistry and Cytochemistry 29, 663-671.

World Health ORganization (1980). Purification of Mycobacterium leprae. Report of the fifth Meeting of the Scientific Working Group on the Immunology of Leprosy (IMMLEP) TDR/IMMLEP-SWG 5 , 80/3 Annex 4, p. 23. Geneva: WHO.

YounG, D. B. (1981). Detection of mycobacterial lipids in skin biopsies from leprosy patients. International Journal of Leprosy 49, 198-204.

Young, D. B. \& BUCHANAN, T. M. (1983). A serological test for leprosy with a glycolipid specific for Mycobacterium leprae. Science 221 , 1057-1059.

Young, D. B., Khanolkar, S. R., Barg, L. L. \& Buchanan, T. M. (1984). Generation and characterization of monoclonal antibodies to the phenolic glycolipid of Mycobacterium leprae. Infection and Immunity 43, 183-188. 